\title{
INTRODUÇÃO À AGRICULTURA DE PRECISÃO: CONCEITOS E VANTAGENS
}

\author{
INTRODUCTION THE PRECISION FARMING: CONCEPTS AND ADVANTAGES
}

\author{
Mauro Tschiedel $^{1}$ Mauro Fernando Ferreira ${ }^{2}$
}

- REVISÃO BIBLIOGRÁFICA -

RESUMO

A revisão bibliográfica apresentada é uma síntese de diversos autores, os quais proporcionam uma introdução à agricultura de precisão, através de conceitos básicos e vantagens que o sistema oferece, demonstrando que não é somente uma colhedora automotriz com um sistema de posicionamento global. A agricultura de precisão pode ser considerada como um amplo conceito, englobando tecnologias e novos conhecimentos de informática, eletrônica, geoprocessamento entre outros. Este conceito incorpora um grande número de conhecimentos científicos novos e alta tecnologia, apresentando ao produtor novos termos, conceitos, equipamentos e tecnologias.

Palavras-chave: agricultura de precisão, SIG, GPS.

\section{SUMMARY}

The presented bibliographical revision is a synthesis of several authors, which provide an introduction to the precision farming, through basic concepts and advantages that the system offers, demonstrating that it is not only a combine harvester with a global positioning system. The precision farming can be considered as a wide concept, including technologies and new computer science knowledge, electronics, geoprocessing among others. This concept incorporates a great number of new scientific knowledge and high technology, presenting to the farmer, new concepts, equipments and technologies.

Key words: precision farming, GIS, GPS.

\section{INTRODUÇÃO}

Há a necessidade do aumento da eficiência de todos os setores da economia globalizada para manter a competitividade. Para a agricultura, não poderia ser diferente. A evolução da informática, tecnologias em geoprocessamento, sistemas de posicionamento global e muitas outras tecnologias estão proporcionado à agricultura uma nova forma de se enxergar a propriedade, deixando de ser uma somente e sim várias propriedades dentro da mesma, porém com características específicas. Esta mudança na forma de fazer agricultura está tornando cada vez mais o produtor rural um empresário rural, por controlar cada vez mais a linha de produção.

Esta mudança é necessária para que se entenda a propriedade não homogênea e sim que se trate cada parte conforme as suas necessidades, fazendo com que o produtor tenha o conhecimento detalhado em cada parte da linha de produção ou cada metro quadrado da sua propriedade.

O objetivo desta revisão é o de trazer os conceitos sobre agricultura de precisão e as suas diferenças entre os diversos autores, bem como as vantagens que o sistema oferece.

\footnotetext{
${ }^{1}$ Aluno do Curso de Agronomia. Departamento de Engenharia Rural (DER), Faculdade de Agronomia Eliseu Maciel (FAEM), Universidade Federal de Pelotas (UFPel). Campus Universitário. CP 354. 96001-970. Capão do Leão - RS. Tel.: (0xx53) 275-7259. E-mail: mtschiedel@bol.com.br. Autor para correspondência.

${ }^{2}$ Engenheiro Agrícola. Mestre. DER/FAEM/UFPel. E-mail: maurof@ufpel.tche.br. 


\section{CONCEITOS E VANTAGENS}

A agricultura de precisão como é chamado no Brasil o sistema de produção adotado por agricultores de países de tecnologia avançada, denominado por eles de Precision Agriculture, Precision Farming, Site-Specific Crop Management (MANZATTO et al., 1999).

A solução hoje utilizada é a de enfocar grandes áreas e entendê-las como homogêneas, levando ao conceito da necessidade média para a aplicação dos insumos - fertilizantes, defensivos, água, etc - o que faz com que, por exemplo, a mesma formulação e/ou quantidade do fertilizante seja utilizada para toda a área, atendendo apenas as necessidades médias e não considerando, desta forma, as necessidades específicas de cada parte do campo. O mesmo acontece para os demais insumos, causando como resultado uma lavoura com produtividade não uniforme (CAPELLI, 1999).

A agricultura de precisão promete reverter o quadro atual permitindo a aplicação de insumos agrícolas nos locais corretos e nas quantidades requeridas (FATORGIS, 1998).

A agricultura de precisão é uma filosofia de gerenciamento agrícola que parte de informações exatas, precisas e se completa com decisões exatas. Agricultura de precisão, também chamada de AP, é uma maneira de gerir um campo produtivo metro a metro, levando em conta o fato de que cada pedaço da fazenda tem propriedades diferentes (ROZA, 2000).

Segundo MANZATTO et al. (1999), o principal conceito é aplicar os insumos no local correto, no momento adequado, as quantidades de insumos necessários à produção agrícola, para áreas cada vez menores e mais homogêneas, tanto quanto a tecnologia e os custos envolvidos o permitam.

Agricultura de precisão combina as novas tecnologias sustentando a era da informação com uma agricultura industrial madura. É um sistema de manejo de produção integrado, que tenta igualar o tipo e a quantia de insumos que entram na propriedade com as necessidades da cultura em pequenas áreas dentro de um campo da propriedade. Esta meta não é nova, mas novas tecnologias agora disponíveis permitem o conceito de agricultura de precisão ser percebido em uma produção prática (DAVIS, 1998).

MIRANDA (1999) dando uma posição mais defensiva quanto à agricultura de precisão menciona que ela envolve um complexo processo, cujo fundamento é o conhecimento espacial preciso da atividade agrícola, frequientemente baseado no uso de dados obtidos com auxílio de satélites.
Industriais e vendedores de insumos, sob argumentos de eficiência, vêem nessa proposta uma nova oportunidade de venda de equipamentos e produtos.

Segundo BATCHELOR et al. (1997) a agricultura de precisão é uma filosofia de manejo da fazenda na qual os produtores são capazes de identificar a variabilidade dentro de um campo, e então manejar aquela variabilidade para aumentar produtividade e os lucros.

O termo agricultura de precisão engloba o uso de tecnologias atuais para o manejo de solo, insumos e culturas, de modo adequado às variações espaciais e temporais em fatores que afetam a produtividade das mesmas (EMBRAPA, 1997).

A agricultura de precisão é a tecnologia cujo objetivo consiste em aumentar a eficiência, com base no manejo diferenciado de áreas na agricultura. A agricultura de precisão não consiste simplesmente na habilidade em aplicar tratamentos que variam de local para local, porém, ela deve ser considerada com a habilidade em monitorar e acessar a atividade agrícola, precisamente em um nível local, tanto que as técnicas de agricultura de precisão devem ser compreendidas como uma forma de manejo sustentável, na qual as mudanças ocorrem sem prejuízos para as reservas naturais, ao mesmo tempo em que os danos ao meio ambiente são minimizados. Além de útil à agricultura de precisão, esta definição engloba a idéia de compromisso no uso da terra, relativamente às gerações futuras. Um manejo sustentável implica algo mais além da manutenção dos índices de produtividade (MANTOVANI $\boldsymbol{e} \boldsymbol{e} \boldsymbol{a l}$., 1998).

DAVIS (1998) afirma que a agricultura de precisão foi freqüentemente definida com base nas tecnologias que permitem que ela seja realizada como GPS (Sistema de Posicionamento Global) ou sistemas de taxa variável. Tão importantes quanto os dispositivos usados na agricultura de precisão, é perceber que a informação usada ou coletada é o ingrediente chave para o sucesso do sistema. O conceito de agricultura de precisão se distingue da agricultura tradicional por seu nível de manejo. Em vez de administrar uma área inteira como uma única unidade, o manejo é adaptado para pequenas áreas pequenas dentro de um campo.

A agricultura de precisão é um termo aplicado a uma larga ordem de tópicos que se relacionam ao manejo preciso de unidades pequenas de terra em contraste com o manejo tradicional onde um campo inteiro é compreendido como uniforme. Esta oportunidade de se administrar pequenas áreas de terra individualmente ficou possível devido à disponibilidade de sistemas de posicionamento 
globais que podem ser usados para localização exata em terra de equipamentos e máquinas (MILLER \& SUPALLA, 1996).

Conforme CANZIAN et al. (1999), alguns campos podem ser bem uniformes, mas outros apresentam variações no tipo de solo, fertilidade e outros fatores que afetam a produção agrícola. Se a variabilidade do campo puder ser medida e registrada, estas informações poderão ser usadas para otimizar as aplicações em cada ponto, sendo este o novo conceito de agricultura de precisão.

BLACKMORE (1996) considera que o manejo da variabilidade é a chave para o uso efetivo da tecnologia de agricultura de precisão.

BLACKMORE et al. (1994) relatam que a agricultura de precisão é o termo que descreve a meta de aumentar a eficiência do manejo de agricultura, sendo uma tecnologia em desenvolvimento, que modifica técnicas existentes e incorpora novas ferramentas para o administrador utilizar.

Conforme ANTUNIASSI (1998), o mapeamento detalhado dos fatores de produção e aplicação localizada de insumos são os princípios básicos do sistema.

Para SEARCY (1997) a idéia da agricultura de precisão é saber o solo e características da produção que causam uma produção diferente para cada parte do campo, e aperfeiçoar as entradas de insumos dentro de porções pequenas do campo. A filosofia atrás de agricultura de precisão é aquela de que os insumos (semente, fertilizante, substâncias químicas, etc.) só deveriam ser aplicados conforme as necessidades e que estes sejam mais econômicos para produção.

Ainda SEARCY (1997) considera técnicas de agricultura de precisão que podem melhorar sustentabilidade econômica e ambiental da produção. Na agricultura de hoje, produtores tendem a cultivar cada campo como uma única unidade. Embora eles reconhecem freqüentemente a variabilidade, eles tratam-no como um campo médio, esperando que o que eles introduzem nele, seja adequado para todo o resto.

CAMPO (2000a) considera que agricultura de precisão é o conjunto de técnicas e procedimentos que permite conhecer, localizar geograficamente e delimitar áreas de diferente produtividade, através do emprego da informática, programas específicos, sensores, controladores de máquinas e sistema de posicionamento global (GPS).

Segundo PARKIN \& BLACKMORE (1995), a agricultura de precisão é uma aproximação de sistemas e requer uma compreensão dos processos envolvidos para alcançar uma meta particular. A meta pode não ser necessariamente maximizar rendimento mas pode ser maximizar vantagem financeira dentro de uma série de constrangimentos ambientais e financeiros.

Para DOERGE (1999) a agricultura de precisão é controlar e colecionar informações agronômicas para prover necessidades atuais de partes de campos em lugar de necessidades comuns para campos inteiros. Aplicação de insumos em locais específicos comumente usada dividindo-se os campos inteiros em zonas de manejo menores, homogêneas.

Para REETZ \& FIXEN (1999) as tecnologias de agricultura de precisão sempre demandam ferramentas agronômicas de produção mais desenvolvidas. $O$ sucesso da sua aplicação depende do entender agronômico do sistema de produção que se administra. Nesse caso, o conhecimento agronômico e bom senso fazem a diferença.

DALLMEYER \& SCHLOSSER (1999) relatam que a agricultura de precisão engloba o uso de tecnologias atuais para o manejo do solo, insumos e culturas de modo adequado para as variações espaciais e temporais nos fatores que afetam a produtividade das mesmas. O que tem levado a esta nova filosofia de prática agrícola é o uso de três novas tecnologias, que são o sensoriamento remoto, o uso de sistemas de informações geográficas (SIG) e o sistema de posicionamento global (GPS). A agricultura de precisão é um conceito de sistema de produção agrícola que envolve o desenvolvimento e a adoção de técnicas de gestão, baseado no conhecimento com o objetivo principal de otimizar a rentabilidade. Este sistema permite práticas de gerenciamento com computador pessoal, que é a possibilidade de administrar cada local do campo adequadamente, se é econômico e tecnicamente vantajoso administrá-lo a este nível.

LOWENBERG-DEBOER (1996) considera a agricultura de precisão uma tecnologia infantil. Segundo ele, esta criança tem alguns sinais da eventual grandeza, mas suas totais capacidades não serão evidentes durante alguns anos. Como todas as crianças, a agricultura de precisão exigirá um investimento de tempo e recursos para ajudar a encontrar a sua maturidade.

Segundo CAPELLI (1999) agricultura de precisão apresenta as vantagens de possibilitar um melhor conhecimento do campo de produção, permitindo, desta forma a tomada de decisões melhor embasadas. Com isto tem-se uma maior capacidade e flexibilidade para a distribuição dos 
insumos naqueles locais e no tempo em que são mais necessários, minimizando os custos de produção; a uniformidade na produtividade é alcançada pela correção dos fatores que contribuem para sua variabilidade obtendo-se, com isto, um aumento global da produtividade; a aplicação localizada dos insumos necessários para sustentar uma alta produtividade contribui com a preservação do meio ambiente, já que estes insumos são aplicados somente nos locais, quantidades e no tempo necessário.

Conforme citado por GENTIL \& FERREIRA (1999) a agricultura de precisão promete grandes benefícios para os usuários deste sistema como:

- redução do grave problema do risco da atividade agrícola;

- redução dos custos da produção;

- tomada de decisão rápida e certa;

- controle de toda situação, pelo uso da informação;

- maior produtividade da lavoura;

- mais tempo livre para o administrador; e

- melhoria do meio ambiente pelo menor uso de defensivo.

Para BATCHELOR et al. (1997) a agricultura de precisão pode:

- melhorar os rendimentos de colheita e lucros;

- fornecer informações para tomar decisões de manejo mais embasadas;

- provêr registros de fazenda mais detalhados e úteis;

- reduzir custos de fertilizante;

- reduzir custos de praguicida; e

- reduzir poluição.

Ainda CAMPO (2000b) atribui à agricultura de precisão, os seguintes benefícios:

- redução de quantidades de insumos;

- redução dos custos de produção;

- redução da contaminação ambiental; e

- aumento no rendimento das culturas.

\section{CONCLUSÕES}

A agricultura de precisão tende a se tornar cada vez mais comum nas propriedades rurais. As tecnologias hoje existentes já permitem que se tenha um grande conhecimento das variabilidades encontradas entre as diferentes áreas da propriedade, o que já proporciona a tomada de decisões com base em dados mais precisos.

A introdução do conceito de agricultura de precisão em propriedades onde se tem como objetivo, maximizar os lucros e minimizar os danos ambientais é imprescindível. Com o advento deste conceito, Ter-se-á um controle maior sobre as possíveis causas na redução da produtividade e/ou dano ambiental.
A agricultura de precisão necessita de um maior número possível de informações para que possa ser implantada com sucesso, como demonstrado na revisão acima.

\section{REFERÊNCIAS BIBLIOGRÁFICAS}

ANTUNIASSI, U.R. Agricultura de precisão: aplicação localizada de agrotóxicos. In: GUEDES, J.V.C.; DORNELES, S.H.B. (Org.) Tecnologia e segurança na aplicação dos agrotóxicos - novas tecnologias. Santa Maria : Departamento de Defesa Fitossanitária; Sociedade de Agronomia de Santa Maria, 1998. p.53-63.

BATCHELOR, B.; WHIGHAM, K.; DEWITT, J., $\boldsymbol{e} t \boldsymbol{a l}$ Precision agriculture: introduction to precision agriculture. Iowa Cooperative Extension, 1997. 4p. Disponível na Internet. http:// www.extension.iastate.edu/ Pages/precisionag/prec-ag.pdf em 18 Ago. 1999.

BLACKMORE, B.S.; WHEELER, P.N.; MORRIS, R.M., et al. The role of precision farming in sustainable agriculture: a European perspective. Minneapolis/USA, 1994. P.13. Disponível na Internet. - http://www.silsoe.cranfield.ac.uk/ cpf/papers/pfsusag2/pfsusag2.pdf em 22 Jul. 1999. Trabalho apresentado na 2 nd International Conference on Site-Specific Management for Agricultural Systems in Minneapolis/USA.

BLACKMORE, B.S. An information system for precision farming. Silsoe. Inglaterra : The Centre for Precision Farming. Cranfield University, 1996. p.09. Disponível na Internet. http://www.silsoe.cranfield.ac.uk/cpf/papers/ ISPF/ispf3.pdf. em 22 Jul. 1999. Trabalho apresentado em the Brighton Conference Pests and Diseases 1996.

CAMPO, P. do. Agricultura de precisão. Inovações do campo. Piracicaba. 2000a. Disponível na Internet. http://www1.portaldocampo.com.br/inovacoes/agric_precisao .htm em 06 Mai. 2000(a).

CAMPO, P. do. Agricultura de precisão. Inovações do campo. Piracicaba. 2000b. Disponível na Internet. http://www1.portaldocampo.com.br/inovacoes/agric_precisao 03.htm em 06 Mai. 2000(b).

CANZIAN, E.; SARAIVA, A.M.; CUGNASCA, C.E., $\boldsymbol{e} \boldsymbol{t}$ al . Projeto de um monitor de semeadora com GPS para pesquisa em agricultura de precisão. Disponível na Internet. http://www.pcs.usp.br/ laa/projetos.html em 27 Jul. 1999.

CAPELLI, N.L. Agricultura de precisão - Novas tecnologias para o processo produtivo. LIE/DMAQAG/ FEAGRI/UNICAMP, 1999. Disponível na Internet. http://wwwbases.cnptia.embrapa.br/cria/gip/gipap/capelli.doc em 15 Out. 1999

DALLMEYER, A.U.; SCHLOSSER, J.F. Mecanizacion para la agricultura de precisión. In: BLU, R. O.; MOLINA, L. F. Agricultura de precisión - Introducción al manejo sitioespecífico. Chillán-Chile : INIA, 1999. Cap.3, p.75-104.

DAVIS, G., CASADY, W.: MASSEY, R Precision agriculture: An introduction. Water quality. University of MissouriSystem, 1998. P.8. Disponível na Internet. http://www.fse.missouri.edu/mpac/pubs/wq0450.pdf em 17 Jun. 1999. 
DOERGE, T.A. Management zone concepts. Site-specific management guidelines. 1999. p.4. Disponível na Internet. http://www.farmresearch.com/SSMG/ssmg-02/SSMG 02.pdf em 04 Dez. 1999.

EMBRAPA. Tecnologia em mecanização no Brasil: Equipamentos e sistemas para o futuro. In: SEMINÁRIO TEMÁTICO PARA PROSPECÇÃO DE DEMANDAS EM PESQUISA E DESENVOLVIMENTO EM MECANIZAÇÃO AGRÍCOLA NO BRASIL, 1997, Sete Lagoas-MG. Disponível na Internet. http://wwwbases.cnptia.embrapa.br/cria/gip/gipap/ seminario.doc em 15 Out. 1999.

FATORGIS. Agricultura de precisão: A tecnologia de GIS/GPS chega às fazendas. Curitiba, 1998. Disponível na Internet. http://www.fatorgis.com em 19 Jul. 1999.

GENTIL, L.V.; FERREIRA, S.M. Agricultura de precisão: Prepare-se para o futuro, mas com os pés no chão. Revista A Granja, Porto Alegre, n 610, 1999. p12-17.

LOWENBERG-DEBOER, J. Economics of precision farming: payoff in the future. Purdue University, 1996. Disponíve na Internet. http://dynamo.ecn.purdue.edu/ biehl/ SiteFarming/economic_issues.html em 28 Abr. 2000.

MANTOVANI, E.C.; QUEIROZ, D.M.; DIAS, G.P. Máquinas e operações utilizadas na agricultura de precisão. In: SILVA, F. M. da.(Coord.). Mecanização e agricultura de precisão. Poços de Caldas : UFLA/SBEA, 1998. p.109-157.

MANZATTO, C.V.; BHERING, S.B.; SIMÕES, M. Agricultura de precisão: propostas e ações da Embrapa solos. EMBRAPA Solos, 1999. Disponível na Internet. http://www.cnps.embrapa.br/search/pesqs/proj01/proj01.html em 01 Out. 1999.
MILLER, W.; SUPALLA, R. Precision farming in Nebraska: A status report, 1996. Disponível na Internet. http://ianrwww.unl.edu/pubs/farmmgt/nf305.htm em. 21 Ago. 1999.

MIRANDA, E.E. de. Informática brasileira em análise. Quem precisa da agricultura de precisão?. Informática Brasileira em Análise. EMBRAPA Monitoramento por Satélite. Disponível na Internet. http://www.cesar.org.br/analise/n_20/ artigon_20.html em 15 Out. 1999.

PARKIN, C.S.; BLACKMORE, B.S. A precision farming approach to the application of agrochemicals. 1995. p.7 Disponível na Internet. http: //www.silsoe.cranfield.ac.uk/ cpf/papers/BAAS/BAAS. pdf em 27 Jul.1999. Trabalho apresentado na British Association for the Advancement of Science meeting, University of Newcastle-upon-Tyne, September 12th, 1995.

REETZ, H.F.Jr.; FIXEN, P.E. Strategic approach to site-specific systems. Site-specific management guidelines. 1999. Disponível na Internet. http://www.farmresearch.com/ SSMG/ssmg-28/SSMG 28.pdf em 04 Dez. 1999.

ROZA, D. Novidade no campo: Geotecnologias renovam a agricultura. Revista InfoGEO, n 11 - jan/fev, 2000. Disponível na Internet. http://www.infogeo.com.br/Revista/ materia_11.htm em 21 Mai. 2000.

SEARCY, S.W. Precision farming: A new approach to crop management. Texas Agricultural Extension Service. The Texas A\&M University System, 1997. p.4. Disponível na Internet. http://agpublications.tamu.edu/pubs/eengine/15177. pdf em 22 Mar. 2000. 